\title{
ALOCAÇÃO DE FLUXOS DE PASSAGEIROS EM UMA REDE DE TRANSPORTE PÚBLICO DE GRANDE PORTE FORMULADO COMO UM PROBLEMA DE INEQUAÇÕES VARIACIONAIS
}

\author{
Fernando Ramiro Castro Aragón \\ José Eugenio Leal * \\ Departamento de Engenharia Industrial \\ PUC-Rio \\ Rio de Janeiro - RJ \\ jel@rdc.puc-rio.br \\ * Corresponding author/autor para quem as correspondências devem ser encaminhadas \\ Recebido em 03/2001, aceito em 03/2003 após 1 revisão
}

\begin{abstract}
Resumo
As grandes metrópoles do mundo enfrentam-se diariamente com problemas de transporte. Para planejar o sistema os tomadores de decisão necessitam de instrumentos capazes de simular, com uma boa aproximação, o processo de escolha de caminhos de todos os usuários nas suas viagens. O trabalho apresenta a formulação e implementação de um modelo de alocação de fluxos de passageiros, sobre uma rede de transporte público. O modelo foi formulado como um problema de inequação variacional. O algoritmo de solução considera algumas hipóteses sobre o processo de partição de fluxos pelos trechos de linhas alternativas. O procedimento foi implementado usando a linguagem Delphi e aplicado em testes com redes divulgadas na literatura. Um grande experimento foi feito com a rede de transporte da cidade do Rio de Janeiro.
\end{abstract}

Palavras-chave: redes de transporte público; alocação de fluxos; modelos de redes.

\begin{abstract}
The biggest cities in the world faced problems in the transportation system. In order to plan the system, decision makers need tools, which enable them to make good approximate simulations of the routing choice process for all the users. The work presents the formulation and implementation of a passenger flow assignment model for a transit transportation network. The model has been stated as a variational inequality problem. The solution algorithm makes some assumptions about the process of flow partition between alternative line segments. The procedure has been implemented using Delphi language and applied in tests with small networks published in the literature. A bigger experiment has been conducted with the transit network of Rio de Janeiro city.
\end{abstract}

Keywords: public transportation networks; flow assignment; network models. 


\section{Introdução}

As grandes metrópoles do mundo enfrentam-se diariamente como problemas de transporte. $\mathrm{Na}$ maioria das cidades, o principal meio de transporte usado pelas pessoas nos seus deslocamentos diários é o público. Trata-se de um sistema bastante complexo cujos principais componentes são o ônibus, o trem urbano e o metrô. Dependendo da cidade, faz-se uso de outros modos como veículos leves sobre trilhos, lotações, vans, táxis coletivos, etc. Os tomadores de decisão tratam com diversos agentes que disputam o mercado representado pela demanda da população. Decisões, com impactos importantes sobre a qualidade de vida das populações, são tomadas sem que exista algum conhecimento prévio das suas conseqüências. Criação ou extinção de linhas, mudanças de traçado, de freqüência, ou de tecnologia produzem efeitos sobre a distribuição de fluxos de passageiros sobre os trechos e veículos do transporte público e interferem no tempo de viagem, no conforto e no número de transferências dos usuários. Modelos de alocação de fluxos servem para prever como os fluxos de passageiros se distribuem sobre a rede de transporte público e deveriam ser instrumentos auxiliares nas tomadas de decisão das autoridades de transportes. Este trabalho tem como objetivo discutir diversas abordagens de modelos de alocação de fluxos em redes de transporte público e detalhar a formulação matemática de um modelo de alocação baseada em inequações variacionais.

O trabalho apresenta na seção dois a conceituação do problema. Na seção três uma revisão bibliográfica descreve o estado da arte partindo dos primeiros modelos dos anos 70 até a atualidade. A seção quatro apresenta a formulação matemática proposta, a seção cinco, a implementação computacional, e a seção seis mostra resultados de experimentos com uma rede de grande porte da região metropolitana do Rio de Janeiro. Finalmente a seção sete apresenta conclusões sobre os resultados da implementação do modelo e sobre possíveis desdobramentos desta pesquisa.

\section{Definição do problema}

Nos problemas de alocação de fluxo de veículos particulares em redes viárias urbanas, existe uma equivalência direta entre os deslocamentos dos usuários (condutores, ou passageiros) e os deslocamentos dos próprios veículos. A alocação de fluxo dos usuários está relacionada diretamente com a determinação dos itinerários dos veículos. No caso do problema de alocação de passageiros de uma rede de transporte coletivo, esta equivalência não existe: os deslocamentos dos ônibus ou os trechos do metrô, em geral não correspondem aos deslocamentos dos usuários. No caso mais geral, os usuários do sistema de transporte coletivo têm que acessar a rede, ou seja têm que se deslocar desde suas origens até uma parada de ônibus, ou uma estação de metrô ou de trem ou de barca, onde esperarão para serem atendidos por algum veículo, e depois saem da rede de transporte público para se deslocar a pé até seus destinos. Os correspondentes tempos de espera são, de modo geral, aleatórios, se é aceita a premissa de que as chegadas dos passageiros e dos veículos são aleatórias. Tudo isto afeta a abordagem do problema.

Então se pode afirmar que em redes de transporte coletivo se faz alocação de passageiros e em redes de transporte particular se alocam veículos. Mesmo assim, as primeiras pesquisas que apareceram na literatura propunham tratar a alocação de passageiros como uma variação do problema de alocação de fluxo de veículos em redes de transporte particular, para os quais já existiam métodos de alocação baseados nos algoritmos desenhados por Moore (1957) e 
Dijsktra (1959), que resolvem o problema de rotas mínimas. Zhan \& Noon (1998) analisaram diversos algoritmos que resolvem o problema de achar rotas mínimas em redes reais. Eles consideram que pesquisas anteriores avaliam o desenvolvimento computacional e a eficiência da implementação dos algoritmos para achar as rotas mínimas, principalmente em base a redes geradas aleatoriamente. Indicam que os métodos para gerar as redes aleatórias variam consideravelmente, e que podem identificar-se diferenças com as redes reais, pelo grau de conectividade, estimado pela razão que mede a incidência arco-nó. As irregularidades na conectividade das redes aleatórias podem favorecer certos tipos de algoritmos na avaliação do desempenho.

Por outro lado, o problema de alocação de equilíbrio em redes de transporte coletivo é abordado pelos diversos modelos existentes na literatura, como um problema estático. Em geral esses modelos visam determinar o estado médio do sistema durante o período estudado, geralmente horas de pico, ou seja determinar as cargas médias na rede e os custos médios dos deslocamentos dos pares origem-destino. Gendrau (1984) justificava isto, explicando que devido ao tamanho das redes estudadas, o estudo do estado médio do sistema nas horas de pico representa, provavelmente, um conjunto de informações suficientes para a tomada de decisões dos planejadores da rede estudada, e que considerar um modelo mais detalhado poderia originar um modelo de tamanho e complexidade que tornaria inviável sua utilização num problema real.

\section{Modelos de alocação: $O$ estado da arte}

Os modelos de alocação de passageiros em redes de transporte coletivo têm experimentado um desenvolvimento importante nas últimas quatro décadas. Os modelos que primeiro aparecem na literatura (Dial, 1967; Andréasson, 1976; Lampkin \& Saalmans, 1967; Hasselströem, 1981), propunham técnicas de alocação similares às utilizadas nos problemas de alocação de fluxo de veículos particulares. Estes modelos assumem que os passageiros viajam nos caminhos mais curtos entre suas origens e destinos, e a maioria não considera os efeitos do problema do congestionamento, sendo chamados de "modelos sem restrição de capacidade".

De modo geral, os modelos que não consideram restrição de capacidade, dependem das hipóteses adotadas acerca do comportamento dos passageiros. O comportamento dos usuários do transporte coletivo na hora de fazer a escolha da rota pode ser classificado em dois tipos segundo a literatura existente: no tipo I o passageiro espera um ônibus de uma linha determinada, e no tipo II o passageiro espera o primeiro ônibus a aparecer no ponto de parada, de um subconjunto de linhas consideradas atrativas.

No primeiro grupo estão aqueles que se baseiam no conceito de rota mínima, ou seja, o usuário do sistema de transporte coletivo utilizará a rota que minimize seu custo generalizado. O usuário já sabe qual é a linha que tem o menor custo generalizado para chegar até seu destino, e somente espera um ônibus daquela linha, o que corresponderia a uma alocação do tipo "tudo ou nada". No segundo grupo, estão aqueles modelos que se fundamentam na idéia de estratégia ótima, conceito formulado por Spiess (1983), que basicamente consiste em escolher um subconjunto de linhas de modo a minimizar o valor esperado do custo total de viagem. Bouzaïene-Ayari et al. (1995) indicam que o que determina uma estratégia de viagem são o conjunto de linhas atrativas escolhidas pelo passageiro em diferentes paradas e os pontos de desembarque daquelas linhas, para 
transbordo ou para caminhada até o destino. Knoppers (1995) ressalta que os transbordos reduzem a atratividade do transporte público como uma alternativa ao carro. A resistência ao transbordo pode ser expressa como o tempo necessário para o transbordo e uma probabilidade de perder a conexão. Conexões perdidas implicam em tempos de espera longos.

De Cea et al. (1990) salientam que se bem os modelos baseados no conceito de estratégia permitem obter uma maior dispersão de fluxos na rede de transporte público, a abordagem da alocação a rotas mínimas é mais eficiente em termos de tempo de cálculo computacional. A existência de seções do caminho que podem ser atendidas por mais de uma linha de ônibus, origina várias opções para escolha dos passageiros, e esta escolha frequientemente não é simples de ser modelada.

Um passageiro, para viajar de um ponto a outro da rede, pode escolher de um conjunto de linhas disponíveis. Umas linhas dentro deste conjunto podem ser muito mais lentas que outras. Chriqui (1974) apresenta um método de determinação do subconjunto das linhas atrativas. Ele parte da idéia de que o subconjunto de linhas é escolhido de modo a minimizar o valor esperado do custo total de viagem, incluindo tempo de espera e tempo em veículo. A linha mais rápida está sempre nesse subconjunto. As outras linhas são ordenadas em forma crescente com respeito ao tempo de viagem em veículo, e somente são incluídas aquelas que diminuem o valor esperado de tempo total de viagem. Chriqui \& Robillard (1975) demonstram que o problema de determinar o subconjunto ótimo de linhas que minimizam o valor esperado do tempo total de viagem, pode ser resolvido como um problema de programação hiperbólica.

Por outro lado, o conceito de rota em uma rede de transporte coletivo não corresponde ao conceito clássico de rota definido para redes de transporte particular ou privado. Os ônibus trafegam pela rede seguindo percursos preestabelecidos. Portanto, os usuários do transporte coletivo escolhem seus caminhos em função das linhas que utilizam e das paradas onde embarcam e desembarcam, para continuar a pé ou fazer um transbordo. Uma rota de transporte coletivo é então definida por uma linha ou combinação de linhas, com os respectivos pontos de embarque e desembarque. Os trechos percorridos na viagem, que serão representados por arcos da rede, aparecem como uma conseqüência do processo de escolha.

Mandl (1978) desenvolve um algoritmo de caminho mais curto apropriado às redes de um sistema de transporte público urbano que, para ele, tem características especiais: os nós desta rede, usualmente representam uma parada ou, de modo mais geral, uma área de uma cidade a ser servida nessa parada. Esta área deveria ser suficientemente pequena para permitir acessar cada ponto dentro dela caminhando desde a parada. Mandl considera, na representação do seu modelo, que a maioria das linhas utiliza os mesmos arcos nos dois sentidos (o que é verdadeiro no caso do metrô e às vezes não é válido para os ônibus) e que a rede viária (que consiste de nós paradas e arcos) é não orientada.

Spiess (1983) propõe um modelo que determina as rotas ótimas em uma rede de transporte coletivo, resolvendo um problema de programação linear para obter as estratégias ótimas de viagem entre dois nós. Ele define uma estratégia como um conjunto de regras que permitem ao passageiro chegar ao seu destino, ou seja, o passageiro vai decidindo sua viagem de acordo com essas regras, podendo ter nós de transbordo, e define a estratégia ótima, como aquela que minimiza o valor esperado do custo total de viagem. Este autor generaliza seu modelo para o caso em que o custo de viagem em veículo em um arco é uma função crescente do fluxo de passageiros naquele arco. 
Nguyen \& Pallottino (1988) definem o conceito de hipercaminho como a representação de uma estratégia, através de um subgrafo acíclico que conecta dois nós da rede, $r$ e $s$, onde $r$ é a origem, $s$ o destino, e onde cada nó $i$ contido no subgrafo, é atravessado por algum caminho que conecta $r$ com $s$, e onde somente existem arcos divergentes nos nós que representam paradas. Isto quer dizer, que para um determinado par origem-destino $r$ - $s$, um hipercaminho representaria uma subrede que contem todas as combinações possíveis de caminhos para ir de $r$ até $s$ (considerando transbordos) utilizando o subconjunto de linhas atrativas para esse par $r$-s. Na Figura 2.1 apresenta-se um exemplo com três paradas, A, B e C e três linhas de ônibus, e na Figura 2.2 mostra-se como seria o hipercaminho que contém todos os caminhos possíveis que conectam A com $\mathrm{C}$.

Modelos desenvolvidos posteriormente começam a considerar a influência do congestionamento. Spiess \& Florian (1989) propõem um modelo que considera que os custos do deslocamento em veículo (custo experimentado pelo passageiro já dentro do ônibus) são funções crescentes do número de passageiros, ou seja, que o efeito do congestionamento representaria o desconforto dos passageiros dentro de um ônibus lotado. Nesse modelo são considerados custos de espera fixos que unicamente dependem da freqüência das linhas, mas que não são afetados pelos efeitos do congestionamento.

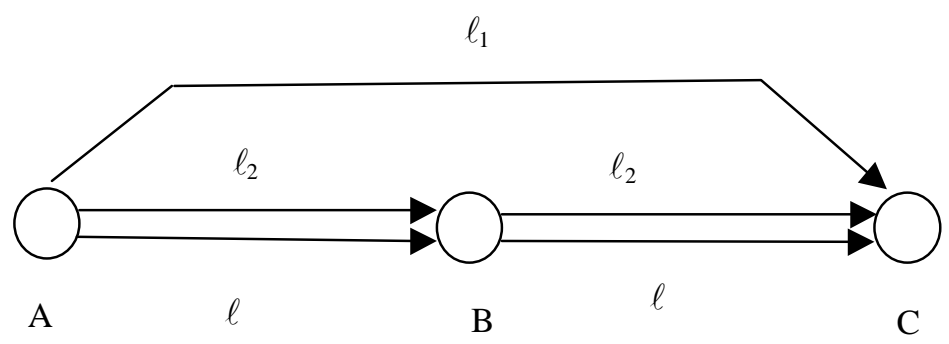

Figura 2.1 - Traçado de três linhas de ônibus

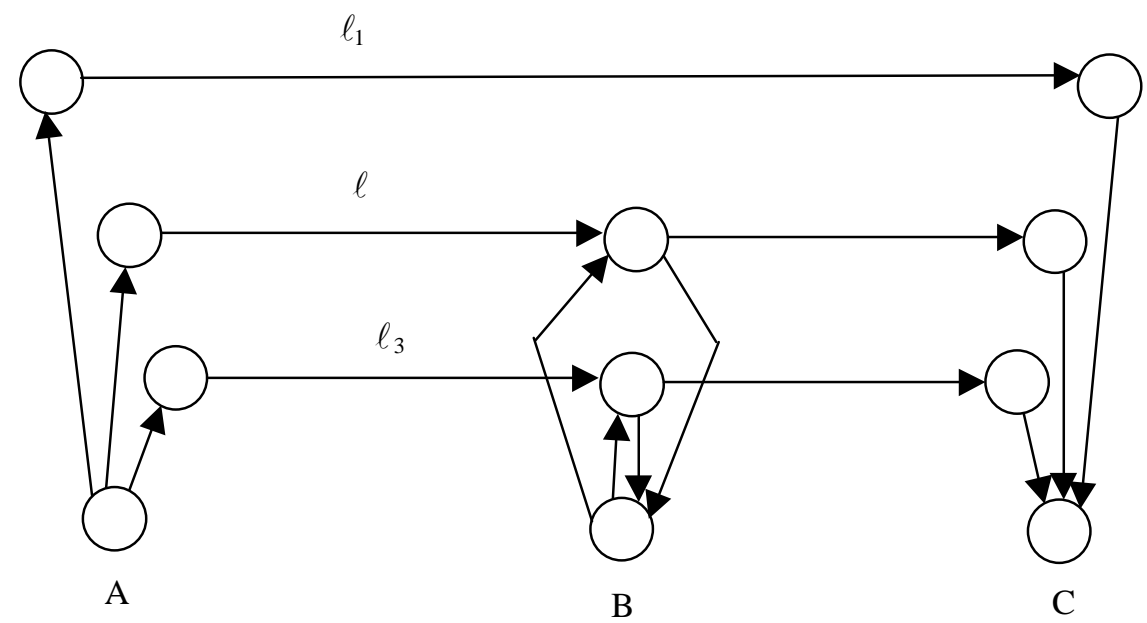


Figura 2.2 - Representação do hipercaminho da figura anterior

Alguns autores consideram os efeitos do congestionamento concentrados nos pontos de parada dos ônibus. Gendrau (1984) considerou efeitos do congestionamento na distribuição de passageiros e nos tempos de espera nas paradas. De Cea \& Fernández (1993-I) e Wu et al. (1994) apresentam formulações em que os passageiros experimentam tempos de espera que dependem da capacidade total da linha (ou conjunto de linhas atrativas) e do fluxo de passageiros nela. De Cea \& Fernández (1993-II) formulam dois modelos em que assumem que os tempos de espera dependem da capacidade dos arcos e do fluxo de passageiros que utilizam as linhas associadas a estes arcos, mas, uma vez que os passageiros abordam um veículo, o tempo de viagem em veículo é fixo, e determinado somente pelo nível de congestionamento na rede viária, o qual é considerado um parâmetro exógeno.

Nguyen \& Pallottino (1986) enfatizam que o problema de alocação de passageiros em redes de transporte coletivo pode ser escrito como um problema de inequação variacional no espaço dos vetores de fluxos nos hipercaminhos viáveis, em forma similar ao problema de inequação variacional no espaço dos vetores de fluxos nos caminhos viáveis para problemas de alocação de tráfego, e que portanto os métodos de solução desenvolvidos para este caso podem ser adaptados para o problema de alocação de passageiros. Wu et al. (1994) formulam um modelo como um problema de inequação variacional, em que os custos de espera dependem da frequiência das linhas e dos efeitos do congestionamento devido às filas de passageiros nas paradas. Muitas modelagens que consideram o fenômeno do congestionamento, formulam o problema como de inequações variacionais em que a função de custo nos arcos tem em geral um Jacobiano assimétrico. Isto quer dizer que o efeito de uma mudança no fluxo do arco $a$, no custo do arco $b$, pode ser diferente do efeito de uma mudança no fluxo do arco $b$, no custo do arco $a$. Matematicamente significa que a derivada parcial do custo no arco $a$ em relação ao fluxo no $\operatorname{arco} b$, não é necessariamente igual à derivada parcial do custo no arco $b$ em relação ao fluxo no $\operatorname{arco} a$.

As redes de transporte público em países em desenvolvimento apresentam algumas características peculiares, como por exemplo, a existência de muitas vias por onde circula uma grande quantidade de linhas de ônibus. A estrutura dos percursos de muitas linhas produz uma grande superposição de ônibus em algumas vias principais. Em muitos países os operadores pressionam para oferecer linhas cujos itinerários atravessam vias com maior demanda. O problema das linhas comuns nas redes de transporte coletivo em algumas cidades de países em desenvolvimento deveria ser modelado considerando a questão do limite de capacidade, o que não é feito em muitos modelos de alocação disponíveis, motivo pelo qual é necessário fazer alguma simplificação.

Os modelos de escolha baseados em função de utilidade, como modelos do tipo Logit, assumem que os passageiros são indivíduos racionais, que associam, a cada alternativa de um conjunto de alternativas possíveis, uma utilidade, e que eles escolhem a alternativa de maior utilidade.

Bunster (1986) indica que nenhuma das diferentes formulações acerca do comportamento dos usuários na hora da escolha da rota, quando existem linhas comuns, pode pretender ser exata, pois na decisão de cada usuário intervém aspectos pessoais que dificilmente podem ser modelados matematicamente. Mas, considerando um comportamento racional e similar em todos os usuários, pode-se supor que o objetivo deles é minimizar o custo generalizado associado à viagem, o que inclui tempo de espera, valor monetário da passagem, tempo de 
deslocamento em veículo, tempo de caminhada, etc. Kirchhoff (1995) considera que informações gerais acerca do serviço de transporte público e acerca das diferentes possibilidades de deslocamento em direção aos destinos dos usuários, são necessárias nas paradas e durante a viagem, para a escolha de rota. Neste contexto são importantes as abordagens que estimam o tempo de viagem experimentado pelos passageiros.

\subsection{Determinação do tempo de viagem}

O modelo de Chriqui (1974) considera que o tempo de viagem entre dois nós da rede A e B, [TTV(A,B)] em que o conjunto de linhas atrativas é L, estará dado pela expressão:

$\operatorname{TTV}(\mathrm{A}, \mathrm{B})=\mathrm{TE}_{\mathrm{L}}+\mathrm{TV}_{\mathrm{L}}(\mathrm{A}, \mathrm{B})$ onde o primeiro termo é o tempo de espera combinado, $\left(\mathrm{TE}_{\mathrm{L}}\right)$ ou seja, o valor esperado do tempo de espera, considerando que o usuário abordará o primeiro veículo de alguma linha do conjunto $\mathrm{L}$ que aparecer na parada, e o segundo termo $\left[\mathrm{TV}_{\mathrm{L}}(\mathrm{A}, \mathrm{B})\right]$ é o valor esperado do tempo em veículo no caso em que o usuário aja da forma mencionada.

Para o caso em que um passageiro pretende ir de A até B utilizando a primeira linha do conjunto L, Chriqui assume que os ônibus das linhas aparecem no ponto de parada A em forma aleatória e independente, e portanto, os tempos de espera de cada linha são variáveis aleatórias independentes, e com distribuições semelhantes. Ele considera também que para cada linha, o tempo de espera de um ônibus é independente do instante de chegada do passageiro ao nó, e apresenta a seguinte propriedade:

$\operatorname{Pr}\left[\operatorname{TE}(\mathrm{i})<=\mathrm{a}_{0}+\mathrm{a} / \mathrm{TE}(\mathrm{i})>\mathrm{a}_{0}\right]=\operatorname{Pr}[\mathrm{TE}(\mathrm{i})<=\mathrm{a}]$, onde TE(i) é o tempo de espera da linha $\mathrm{i}$.

Isto equivale a dizer que o tempo de espera de um ônibus da linha $\mathrm{i}$, não depende do tempo de espera já transcorrido. Esta propriedade de "falta de memória" é encontrada na distribuição exponencial, e Chriqui supõe uma distribuição exponencial para as variáveis aleatórias dos tempos de espera das linhas. Considerando a hipótese de que o passageiro tomará a primeira linha que chegue, ele apresenta a seguinte formulação para o valor esperado do tempo de espera:

$$
\mathrm{E}\left[\mathrm{TE}_{\mathrm{M}}\right]=\int_{0}^{\infty} \mathrm{t} *\left(\sum_{i=1}^{n} 1 / \mathrm{w}_{\mathrm{i}}\right) * \exp \left[-\mathrm{t} \times \sum_{i=1}^{n} 1 / \mathrm{w}_{\mathrm{i}}\right] \mathrm{dt},
$$

onde $\mathrm{w}_{\mathrm{i}}$ é o tempo médio de espera para a linha $\mathrm{i}$.

Integrando por partes chega-se a:

$$
\mathrm{E}\left[\mathrm{TE}_{\mathrm{M}}\right]=1 /\left(\sum_{i=1}^{n} 1 / \mathrm{w}_{\mathrm{i}}\right)
$$

Esta fórmula corresponde ao conceito intuitivo de que o tempo de espera é aquele que o passageiro experimenta ao esperar qualquer linha de um conjunto cuja freqüência total é a soma das freqüências das linhas do conjunto.

Spiess (1983) considera que para uma taxa de chegada uniforme de passageiros ao ponto de parada pode-se considerar que para uma determinada linha $\mathrm{i}, \mathrm{w}_{\mathrm{i}}=\mathrm{a} / \mathrm{F}(\mathrm{i})$, onde a é uma constante positiva e F(i) é a freqüência da linha i (número de veículos / unidade de tempo). A constante assume dois valores: $\mathrm{a}=1$, para uma distribuição exponencial dos headways, com média $1 / \mathrm{F}(\mathrm{i})$ e a = 1/2 como uma aproximação para headways constantes. $\mathrm{O}$ fator a pode 
modelar o efeito de diferentes percepções dos tempos de espera e dos tempos em veículo. Sem perda de generalidade Spiess (1983) e Chriqui (1974) assumem um valor de $\mathrm{a}=1$.

$$
\text { Logo: } \quad \mathrm{E}\left[\mathrm{TE}_{\mathrm{M}}\right]=1 / \sum_{i=1}^{n} \mathrm{~F}(\mathrm{i})
$$

A probabilidade de que a linha i se apresente primeiro é igual a probabilidade de que TE(i) seja t e, para $\mathrm{j} \neq \mathrm{i}, \mathrm{TE}(\mathrm{j})>\mathrm{t}$, ou seja:

$$
\begin{gathered}
\operatorname{Pr}\left[\mathrm{TE}_{\mathrm{M}}=\mathrm{TE}(\mathrm{i})\right]=\int_{0}^{\infty} 1 / \mathrm{w}_{\mathrm{i}} \exp \left[-\mathrm{t} / \mathrm{w}_{\mathrm{i}}\right] * \exp \left[-\mathrm{t} \times \sum_{j \neq i} 1 / \mathrm{w}_{\mathrm{j}}\right] \mathrm{dt} \\
\operatorname{Pr}\left[\mathrm{TE}_{\mathrm{M}}=\mathrm{TE}(\mathrm{i})\right]=\left(1 / \mathrm{w}_{\mathrm{i}}\right) /\left(\sum_{i=1}^{n} 1 / \mathrm{w}_{\mathrm{i}}\right)=\mathrm{F}(\mathrm{i}) / \sum_{i=1}^{n} \mathrm{~F}(\mathrm{i})
\end{gathered}
$$

Se o tempo em veículo pela linha i é TV(i), então o tempo médio em veículo independente da linha tomada será:

$$
\mathrm{TV}=\sum_{i=1}^{n} \mathrm{TV}(\mathrm{i}) \operatorname{Pr}\left[\mathrm{TE}_{\mathrm{M}}=\mathrm{TE}(\mathrm{i})\right]=\mathrm{TV}(\mathrm{i}) * \mathrm{~F}(\mathrm{i}) / \sum_{i=1}^{n} \mathrm{~F}(\mathrm{i})
$$

E o tempo total de viagem esperado será:

$$
\mathrm{TTV}=1 / \sum_{i=1}^{n} \mathrm{~F}(\mathrm{i})+\sum_{i=1}^{n}\left(\mathrm{TV}(\mathrm{i}) *\left(\mathrm{~F}(\mathrm{i}) / \sum_{i=1}^{n} \mathrm{~F}(\mathrm{i})\right)\right)
$$

Acomodando termos:

$$
\mathrm{TTV}=\left(1+\sum_{i=1}^{n} \mathrm{TV}(\mathrm{i}) * \mathrm{~F}(\mathrm{i})\right) / \sum_{i=1}^{n} \mathrm{~F}(\mathrm{i})
$$

Este valor de TTV é válido caso o passageiro tome qualquer primeira linha que o leve até o destino, ou seja, são consideradas todas as linhas que passam na parada e que podem levar o passageiro ao seu destino. No caso geral o passageiro faz uma seleção de linhas atrativas, escolhendo aquelas que não aumentem "muito" o tempo total de viagem. A continuação é descrito o método proposto por Chriqui (1974) para a determinação das linhas comuns ou atrativas.

$\mathrm{O}$ problema de encontrar as linhas comuns equivale a encontrar os valores $\mathrm{X}_{\mathrm{i}}$ tais que minimizem o valor esperado do tempo total de viagem:

$$
\text { Min TTV }=\left(1+\sum_{i=1}^{n} \mathrm{X}_{\mathrm{i}} * \mathrm{TV}(\mathrm{i}) * \mathrm{~F}(\mathrm{i})\right) / \sum_{i=1}^{n} \mathrm{X}_{\mathrm{i}} * \mathrm{~F}(\mathrm{i})
$$

O problema foi formulado como um problema de programação hiperbólica e o procedimento, proposto por Chriqui e denominado algoritmo "S", é descrito a seguir:

Primeiro é definido: $\quad \mathrm{TTV}_{\mathrm{K}}=\left(1+\sum_{i=1}^{k} \mathrm{TV}(\mathrm{i}) * \mathrm{~F}(\mathrm{i})\right) / \sum_{i=1}^{k} \mathrm{~F}(\mathrm{i})$

Etapa 1. Ordenar as linhas com respeito aos tempos em veículos em forma crescente:

$\mathrm{D}=\left\{1_{1}, 1_{2}, \ldots, 1_{\mathrm{n}}\right\}$ e $\mathrm{TV}(1), \mathrm{TV}(2), \ldots, \mathrm{TV}(\mathrm{n})$ com $\mathrm{TV}(1) \leq \mathrm{TV}(2) \leq \ldots \leq \mathrm{TV}(\mathrm{n})$ 
Faça $\quad \mathrm{TTV}_{\mathrm{L}}=\mathrm{TV}_{1}$,

$$
\mathrm{L}=\left\{\mathrm{l}_{1}\right\}
$$

Etapa 2. Faça para $\mathrm{i}=2,3, \ldots, \mathrm{n}$ :

$$
\begin{array}{ll}
\text { Se } \operatorname{TV}(\mathrm{i}) \leq \mathrm{TTV}_{\mathrm{L}} \text { então: } & \mathrm{TTV}_{\mathrm{L}}=\mathrm{TTV}_{\mathrm{i}} \\
\mathrm{L}=\mathrm{L} \cup\left\{1_{1}\right\}
\end{array}
$$

caso contrário parar

\section{Formulação matemática do problema de alocação por equilíbrio em redes de transporte coletivo}

O problema de alocação por equilíbrio em redes de transporte coletivo, abordado neste trabalho, está caracterizado pelas seguintes hipóteses:

- a escolha das rotas dos passageiros está baseada nas estratégias ótimas, representação no contexto dos hipercaminhos;

- a alocação de passageiros é efetuada segundo o primeiro princípio de Wardrop, conhecido como ótimo do usuário;

- a repartição dos passageiros entre os caminhos elementares que compõem um hipercaminho será baseada em um modelo logit;

- a demanda de viagens é considerada fixa, isto quer dizer, a matriz origem-destino tem valores constantes e conhecidos;

- a matriz de custos nos arcos é assimétrica;

- $\quad$ as funções de custos nos hipercaminhos são funções convexas.

Para chegar na formulação do problema que contempla as hipóteses acima indicadas serão apresentadas antes as formulações de um problema de alocação para os seguintes casos:

- um modelo de alocação com custos fixos, no qual os efeitos do congestionamento não são levados em consideração;

- um modelo de alocação com funções de custo no arco não lineares, dependentes do fluxo no próprio arco; e

- $\quad$ um modelo logit de alocação.

\subsection{Modelo de alocação com custos fixos}

Um modelo baseado nas estratégias ótimas para redes de transporte coletivo com custos fixos nos arcos é apresentado brevemente nesta seção (Spiess, 1983).

A rede de transporte coletivo é representada por um grafo $G=(I, A)$, onde os elementos do conjunto de nós, $i \in I$, são conectados por um conjunto de $\operatorname{arcos}$ direcionados $a=(i, j) \in A$. O conjunto de arcos saindo do nó $i$ (correspondendo a uma estrutura forward star) é denotado por $A_{i}^{+}$, e o conjunto de arcos chegando ao nó $i$ (backward star) é denotado por $A_{i}$.

Uma impedância $c_{a}$ e uma frequiência $f_{a}$ são associados a cada arco $a$. No caso de um modelo de alocação com custos fixos, a impedância de cada arco é um valor constante, e na literatura é representado pelo tempo de viagem ou por um custo generalizado de viagem. A demanda de viagens entre os nós $p$ e $q$ está dada por $g_{p q}$. 
Uma rede aumentada, gerada a partir da representação básica da rede de transportes, é utilizada para representar o sistema de transporte coletivo. Um procedimento automatizado da explosão da rede, idealizado e desenvolvido nesta pesquisa, é explicado com detalhe na seguinte seção. Os itinerários das linhas de ônibus, metrô, trens e barcas estão implicitamente contidos na rede básica de transportes. O conjunto de nós da rede aumentada contém, além dos nós físicos da rede básica, um nó adicional por cada parada de cada linha. Correspondentemente, os arcos são subdivididos em vários tipos, como arcos de embarque, arcos em veículo, arcos de desembarque ou descida e arcos de caminhada. Somente os arcos de embarque representam o evento da espera, portanto estes arcos têm uma freqüência finita $f_{a}$. Os outros arcos são atendidos em forma contínua, adotando portanto uma frequiência infinita $\left(f_{a}=\infty\right)$.

O tempo de espera em um nó $i$ depende do conjunto de arcos atrativos $\AA_{i}^{+} \subseteq A_{i}{ }^{+}$, ou seja, depende do subconjunto de arcos saindo de $i$, que são considerados equivalentes em termos de custo pelos usuários. Os passageiros abordarão o primeiro veículo que corresponda a qualquer um destes arcos atrativos. Para um conjunto de arcos atrativos $\AA_{i}^{+}$no nó $i$, o tempo de espera combinado $W\left(\AA_{i}^{+}\right)$é proporcional à freqüência total combinada desses arcos:

$$
W\left(\stackrel{\circ}{i}^{+}\right)=\alpha / \sum_{\mathrm{a} \in \AA_{\mathrm{A}^{+}}^{+}} f_{a}, \quad \alpha>0 \quad(\alpha \text { parâmetro de calibração })
$$

E a probabilidade $P_{a}\left(\AA_{i}^{+}\right)$de passar pelo arco $a$ saindo do nó $i$ é:

$$
P_{a}\left(\AA_{i}^{+}\right)=f_{a} / \sum_{a^{\prime} \in \check{\AA}_{\mathrm{i}}^{+}} f_{a^{\prime}}, \quad a \in \stackrel{\AA}{i}^{+}
$$

Em base a (11) e (12) qualquer estratégia para chegar ao destino $r$ estaria completamente definida pelo correspondente subconjunto de arcos atrativos $\AA \subseteq A$.

A estratégia ótima para chegar ao destino é aquela que minimiza o valor esperado do custo total de viagem. O custo de uma estratégia é a soma dos tempos de viagem dos arcos da estratégia $c_{a}$ ponderados pela probabilidade de viajar por cada arco $a$, e do tempo de espera nos nós $i$ da estratégia, ponderados pela probabilidade de viajar pelo nó $i$.

Para este problema com custos fixos nos $\operatorname{arcos} c_{a}$, a alocação das viagens desde todas as origens até o destino $r$, segundo a estratégia ótima, corresponde a resolver o seguinte problema de otimização linear:

$$
\operatorname{Min} \sum_{a \in A} c_{a} v_{a}+\sum_{i \in I} w_{i}
$$

sujeito a:

$$
\begin{gathered}
\sum_{a \in A_{i}^{+}} v_{a}-\sum_{a \in A_{i}^{-}} v_{a}=g_{i r}, \quad i \in I, \\
v_{a} \leq f_{a} w_{i}, \quad a \in A_{i}^{+}, \quad i \in I, \\
v_{a} \geq 0, \quad a \in A,
\end{gathered}
$$

Aqui $v_{a}$ é o fluxo no arco $a$ e $w_{i}$ representa o tempo total de espera, em pessoas-minutos no nó $i$. 
Este problema pode ser resolvido em forma eficiente utilizando um algoritmo do tipo labelsetting proposto por Spiess (1983), que é apresentado a seguir, no qual são determinadas as estratégias ótimas desde todos os nós da rede até um determinado destino $r$. Para fazer a alocação para todos os destinos, basta executar o algoritmo uma vez para cada destino $r$.

Para chegar ao nó de destino $r$, são achados desde todos os nós de origem: a estratégia ótima $\AA^{*}$ e os valores esperados dos tempos totais de viagem ótimos $u_{i}^{*}$ desde cada nó $i \in I$ até o destino $r$. Isto é feito nos passos 0 a 2 . No passo 3 é realizada a alocação dos fluxos.

Passo 0. Inicialização:

$$
\begin{aligned}
& u_{i} \leftarrow \infty, \quad i \in I-\{r\} ; \quad u_{r} \leftarrow 0 \\
& f_{i} \leftarrow 0, \quad V_{i} \leftarrow g_{i r}, \quad i \in I ; \\
& S \leftarrow A ; \AA \leftarrow \phi
\end{aligned}
$$

Passo 1. Ache o próximo arco:

$$
\begin{aligned}
& \text { se } S=\phi \text { então vá para o passo } 3, \\
& \text { se não, } \\
& S \leftarrow S-\left\{\text { ache } a=(i, j) \in S \text { com o mínimo } u_{j}+c_{a} ;\right.
\end{aligned}
$$

Passo 2. Atualize a etiqueta do nó $i$ :

$$
\begin{aligned}
& \text { se } u_{i} \geq u_{j}+c_{a} \text { então: } \\
& u_{i} \leftarrow\left(f_{j} u_{i}+f_{a}\left(u_{j}+c_{a}\right)\right) /\left(f_{i}+f_{a}\right), \\
& f_{i} \leftarrow f_{i}+f_{a}, \AA \leftarrow \AA+\{a\} \text {; }
\end{aligned}
$$

vá para o passo 1 .

Passo 3. Aloca demanda segundo a estratégia ótima:

Para todo arco $a \in A$ na ordem decrescente de $\left(u_{j}+v_{a}\right)$ :

$$
\begin{array}{ll}
\text { se } a \in \AA & \text { então: } \\
& v_{a} \leftarrow\left(f_{a} / f_{i}\right) V_{i}, \\
& V_{j} \leftarrow V_{j}+v_{a}, \\
\text { se não: } & v_{a} \leftarrow 0
\end{array}
$$

A variável $V_{i}$ representa o fluxo que passa pelo nó $i$. Neste algoritmo a distribuição de passageiros entre as linhas atrativas em cada parada, é feita em forma proporcional às frequiências de cada linha atrativa.

\subsection{Alocação de fluxo em redes de transporte coletivo com funções de custo não-lineares}

Nesta seção é tratado o problema de alocação de fluxo em que os tempos de viagem nos $\operatorname{arcos} c_{a}$ não são constantes, mas são funções contínuas não-decrescentes $c_{a}\left(v_{a}\right)$ do fluxo total no correspondente arco $a$. Essa dependência dos custos nos arcos em função dos volumes de passageiros pode representar uma diminuição da rapidez do veículo devido ao número de passageiros, mas poderia também ser interpretado como um custo generalizado que inclui um termo do desconforto que aumenta a medida que o veículo vai ficando lotado.

As principais hipóteses deste modelo são as seguintes: 
- todos os passageiros que viajam nos veículos sofrem o mesmo grau de desconforto, mesmo que aqueles passageiros que abordaram o veículo nas paradas anteriores tenham maiores chances de conseguir um assento;

- os tempos de espera não são afetados diretamente pelo volume de passageiros. Os passageiros podem abordar o primeiro veículo que chegar na parada pois a capacidade dos veículos não é considerada limitada.

Se $v_{a}{ }^{r}$ representa o volume de passageiros no arco $a \in A$ cujo destino é o nó $r \in R$, então o volume total de passageiros no arco $a$ será a soma dos volumes $v_{a}$ para todos os diferentes destinos $r \in R$,

$$
v_{a}=\sum_{r \in R} v_{a}^{r}, \quad a \in A
$$

Neste contexto, o problema de alocação em uma rede de transporte coletivo não é separável por nó de destino devido a que o custo em cada arco depende do fluxo total de passageiros que trafegam pelo arco.

Se a demanda de viagens desde o nó $i \in I$ até o destino $r \in R$ é $g_{i}{ }^{r}, K_{r}$ o conjunto de todas as estratégias para chegar ao destino $r, h_{i}^{k}$ a parte da demanda $g_{i}^{r}$ que é alocada à estratégia $k \in K$, a seguinte equação garantirá a conservação do fluxo:

$$
\sum_{\mathrm{k} \in K_{r}} h_{i}^{k}=g_{i}^{r}, \quad i \in I, \quad k \in K_{r}, \quad r \in R
$$

Similarmente ao caso em que os custos são constantes, cada passageiro escolhe a estratégia que minimiza o valor esperado do tempo total de viagem, só que neste caso os tempos de viagem dependem dos volumes totais nos arcos e portanto das estratégias escolhidas pelos outros passageiros. O comportamento dos passageiros pode ser caracterizado matematicamente por condições de equilíbrio similares àquelas que se originam do primeiro princípio de Wardrop, utilizadas em uma alocação de tráfego de equilíbrio em uma rede de carros particulares.

Se $s_{i}{ }^{k}$ representa o custo de viagem esperado desde o nó $i$ até o destino $r$ utilizando a estratégia $k \in K_{r}$, e $u_{i}{ }^{r *}$ representa o mínimo custo esperado de viagem desde o nó $i$ até o destino $r$, as condições de equilíbrio podem ser escritas assim:

$$
\left.\begin{array}{l}
s_{i}^{k^{*}}=u_{i}^{r^{*}}, \text { se } h_{i}^{k^{*}} \geq 0 \\
s_{i}{ }^{k^{*}} \geq u_{i}^{r^{*}}, \text { se } h_{i}^{k^{*}}=0
\end{array}\right\}, \quad i \in I, \quad k \in K_{r}, \quad r \in R
$$

Da expressão (19) pode-se dizer que somente existirá fluxo não nulo nas estratégias cujos custos sejam iguais aos custos da estratégia ótima, e naquelas cujo custo é maior não existirá fluxo, o que implica em que somente as estratégias com o menor custo esperado serão utilizadas pelos passageiros.

Spiess (1983) demonstra que as condições de equilíbrio da equação (19) são a solução ótima do seguinte problema de minimização convexa:

$$
\min \sum_{a \in A} \int_{0}^{v a} c_{a}(x) d x+\sum_{i \in I} \sum_{r \in R} w_{i}^{r}
$$

sujeito a: 


$$
\begin{aligned}
& v_{a}=\sum_{r \in R} v_{a}^{r}, \quad a \in A, \\
& v_{a}^{r} \leq f_{a} w_{i}^{r}, \quad a \in A_{i}^{+}, \quad i \in I, \quad r \in R, \\
& \sum_{A_{i}^{+}} v_{a}^{r}-\sum_{A_{i}^{-}} v_{a}^{r}=g_{i r}, \quad i \in I, \quad r \in R, \\
& v_{a}^{r} \geq 0, \quad a \in A, \quad r \in R,
\end{aligned}
$$

onde $c_{a}(x)$ é a função de custo no arco $a$ que depende do fluxo no próprio arco

$w_{i}^{r}$ é o custo total de espera para ir desde $i$ até $r$

$f_{a}$ é a frequiência do $\operatorname{arco} a$

Spiess ressalta que as restrições (21), (22), (23) e (24) do problema são separáveis por destino (somente as restrições, a função objetivo não), e que portanto é possível reformular o problema em termos dos volumes $v_{a}{ }^{k}$ associados com cada estratégia viável $k \in K$ da seguinte forma:

$$
\min \sum_{a \in A} \int_{0}^{v a} c_{a}(x) d x+\sum_{r \in R} \sum_{k \in K_{r}} \sum_{i \in I} w_{i}^{k}
$$

sujeito a:

$$
\begin{aligned}
& v_{a}{ }^{k}=f_{a} x_{a}{ }^{k} w_{i}{ }^{k}, \quad a \in A_{i}^{+}, \quad i \in I, \quad k \in K_{r}, \quad r \in R, \\
& \sum_{a \in A_{i}^{+}} v_{a}{ }^{k}-\sum_{a \in A_{i}^{-}} v_{a}^{k}=h_{i}^{k}, \quad i \in I, \quad k \in K_{r}, \quad r \in R, \\
& v_{a}=\sum_{i \in I} \sum_{k \in K_{r}} v_{a}^{k}, \quad a \in A, \\
& w_{i}^{k} \geq 0, \quad h_{i}^{k} \geq 0, \quad i \in I, \quad k \in K_{r}, \quad r \in R
\end{aligned}
$$

As constantes $x_{a}{ }^{k}$ são iguais a 1 , se o arco $a$ faz parte da estratégia $k$, e 0 em caso contrário. Se $\underline{m}_{a}{ }^{k}, \underline{s}_{i}{ }^{k}$ e $\underline{u}_{i}^{r}$ são as variáveis duais que correspondem às restrições (26), (27) e (18), as condições de Kuhn-Tucker para uma solução ótima $\left(v^{*}, w^{*}, h^{*}\right)$ do problema (25) podem ser escritas assim:

$$
\left.\begin{array}{l}
\underline{s}_{j}^{k^{*}}+t_{a}\left(v_{a}{ }^{*}\right)+\underline{m}_{a}{ }^{k^{*}}=\underline{s}_{i}^{k^{*}}, \text { se } h_{i}^{k^{*}}>0 \\
\underline{s}_{j}{ }^{*}+t_{a}\left(v_{a}{ }^{*}\right)+\underline{m}_{a}{ }^{k^{*}} \geq \underline{s}_{i}{ }^{k^{*}}, \text { se } h_{i}^{k^{*}}=0
\end{array}\right\} a=(i, j) \in A, k \in K_{r}, r \in R
$$

onde $t_{a}\left(v_{a}{ }^{*}\right)$ é o custo no arco $a$ para o fluxo ótimo $v_{a}$

$$
\sum_{a \in A_{i}^{+}} \underline{m}_{a}{ }^{*} x_{a}{ }^{k} f_{a}=1, \quad i \in I, \quad k \in K_{r}, \quad r \in R
$$

$\mathrm{e}$

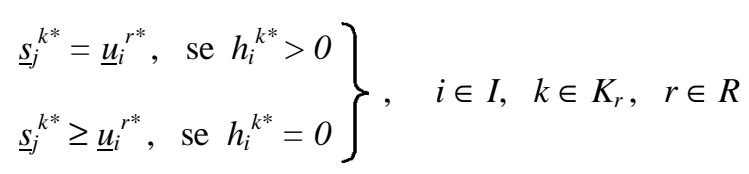


As variáveis $\underline{s}_{i}{ }^{*}$ correspondem ao tempo de viagem esperado desde o nó $i$ até o destino $r$ utilizando a estratégia $k$, isto é:

$$
\underline{s}_{i}^{k^{*}}=s_{i}^{k^{*}}, \quad i \in I, \quad k \in K_{r}, \quad r \in R
$$

Como por (32) $\underline{\boldsymbol{u}}_{i}^{r^{*}}=\min _{k \in K_{r}} \underline{\boldsymbol{s}}_{i}^{k^{*}}$ então:

$$
\underline{u}_{i}^{r^{*}}=u_{i}^{r^{*}}, \quad i \in I, \quad r \in R
$$

o que garante que (32) é equivalente a (19).

Spiess (1983) sugere que este problema pode ser resolvido utilizando um algoritmo baseado no método de Frank \& Wolfe (1956) para sucessivas aproximações lineares da função objetivo. Spiess indica que, dado que não é necessário guardar explicitamente os volumes dependentes dos destinos $v_{a}{ }^{r}$, é possível trabalhar com grandes redes com muitos nós de destino. Uma aproximação linear deste problema origina um novo problema em que é possível separar os fluxos por destino. Para cada destino, o subproblema resultante é equivalente a um problema com custos fixos nos arcos e, portanto, pode ser resolvido utilizando o algoritmo apresentado na seção 4.1.

A continuação é apresentado o algoritmo que resolve o problema de alocação com funções de custo não-lineares.

Passo 0. Inicialização

Ache uma solução viável $\left(v^{0}, w^{0}\right)$ onde $v^{0}$ denota o vetor dos fluxos totais no $\operatorname{arco} a$, e o escalar $w^{0}$ denota o correspondente tempo total de espera: $\sum_{i \in I} \sum_{r \in R} w_{i}^{r}$

$$
l \leftarrow 0
$$

Passo 1. Subproblema

$$
l \leftarrow l+1
$$

calcule $\left(v^{\prime}, w^{\prime}\right)$ resolvendo o problema de alocação com custos fixos $c_{a}=c_{a}\left(v_{a}{ }^{l-1}\right)$ para cada destino $r \in R$.

Passo 2. Busca linear

Calcule o tamanho de passo $\lambda^{1}$, que minimize a função objetivo no segmento de linha determinado por: $(1-\lambda) .\left(v^{l-1}, w^{l-1}\right)+\lambda$. (v', w'), $0 \leq \lambda \leq 1$

Passo 3. Atualização

$$
\left(v^{l}, w^{l}\right) \leftarrow\left(1-\lambda^{l}\right) .\left(v^{l-1}, w^{l-1}\right)+\lambda^{l} .\left(v^{\prime}, w^{\prime}\right)
$$

Se $\sum_{a \in A} c_{a}\left(v_{a}^{l-1}\right)\left(v_{a}^{l-1}-v_{a}{ }^{\prime}\right)+w^{l-1}-w^{\prime}<\varepsilon$ então pare, senão vá ao passo 1 .

A minimização do passo 2 pode ser implementada igualando a zero a derivada da função objetivo. $\mathrm{O}$ que seria equivalente a resolver a seguinte equação:

$$
\sum_{a \in A} c_{a}\left(v_{a}^{l-1}+\lambda\left(v_{a}{ }^{\prime}-v_{a}^{l-1}\right)\right)\left(v_{a}{ }^{\prime}-v_{a}^{l-1}\right)+\lambda\left(w^{\prime}-w^{l-1}\right)=0
$$

Este mesmo problema de uma minimização convexa com custos simétricos, pode ser também formulado como um problema de inequações variacionais, o que é tratado na próxima seção. 


\subsection{Formulação do problema de equilíbrio como um problema de inequações variacionais}

O problema de equilíbrio pode ser escrito como um problema de inequações variacionais, como a continuação se apresenta.

Um problema de inequações variacionais consiste em achar o vetor $x^{*}$ que satisfaça a seguinte expressão:

$$
c\left(x^{*}\right)^{T}\left(x-x^{*}\right) \geq 0
$$

onde $x \in \Omega \subset R^{n}$, um conjunto não vazio, e $c(x)$ é uma função de $R^{n}$ em $R^{n}$.

Por outro lado, um problema de complementaridade não linear, equivalente ao problema de inequações variacionais, é definido por:

$$
\begin{aligned}
& c(x)^{T} x=0 \\
& c(x) \geq 0, \\
& x \geq 0
\end{aligned}
$$

Torres (1987) apresenta a equivalência entre um problema de equilíbrio em uma rede de veículos particulares e um problema de complementaridade não linear. Seguindo a mesma lógica pode-se provar que um problema de equilíbrio para uma rede de transporte coletivo também pode ser expresso como um problema de complementaridade não linear.

Primeiro, considerando que as condições de ótimo do usuário (9) também podem ser escritas como:

$$
\left.\begin{array}{c}
\left(s_{i}{ }^{*}-u_{i}^{r^{*}}\right) h_{i}^{k^{*}}=0 \\
s_{i}^{k^{*}}-u_{i}^{r^{*}} \geq 0
\end{array}\right\}, \quad i \in I, \quad k \in K_{r}, \quad r \in R
$$

e as condições de conservação de fluxo e de não negatividade dos fluxos e das variáveis duais (os multiplicadores das restrições):

$$
\begin{aligned}
\sum_{\mathrm{k} \in K r} h_{i}{ }^{k} & -g_{i}^{r}=0, \quad i \in I, \quad k \in K_{r}, \quad r \in R \\
h & \geq 0 \\
u & \geq 0
\end{aligned}
$$

Pode-se demonstrar que uma solução $x=(h, u)$ que satisfaz as relações $(40)$ - (43) também resolve o problema de complementaridade não linear:

$$
\begin{aligned}
& c(x)^{T} x=0 \\
& c(x) \geq 0, \\
& x \geq 0
\end{aligned}
$$

se é definido apropriadamente $c(x)=(y(x), z(x))$ uma função de $R^{n}$ em $R^{n}$, com $n=/ K /+/ I x R /$ onde $y(x)$ e $z(x)$ são definidas assim:

$$
y_{k}(x)=s_{i}^{k^{*}}-u_{i}^{r^{*}}, \quad i \in I, \quad k \in K_{r}, \quad r \in R
$$




$$
z_{i r}(x)=\sum_{\mathrm{k} \in K r} h_{i}^{k}-g_{i}^{r}, \quad i \in I, \quad r \in R
$$

Então um problema de equilíbrio de redes de transporte coletivo pode ser escrito como um problema de complementaridade não linear e, portanto, também como um de inequações variacionais.

\subsection{Alocação de equilíbrio em redes de transporte coletivo com custos assimétricos}

Nesta seção é tratado o problema de alocação de equilíbrio, quando os custos nos arcos são funções convexas não decrescentes que dependem, não somente do fluxo no próprio arco, senão também dos fluxos em outros arcos da rede.

O algoritmo apresentado na seção 4.2, satisfatório para o caso em que o custo $c_{a}$ em cada $\operatorname{arco} a$ depende somente do fluxo no $\operatorname{arco} a$, não é capaz de resolver situações como as que se apresentam quando os custos nos arcos não dependem unicamente dos fluxos nos mesmos. Exemplos destas situações se apresentam em ruas de mão dupla em que os tempos de deslocamento em cada sentido dependem não somente do fluxo numa direção, senão também na direção contrária. Outro exemplo aparece nas interseções, em que o tempo de deslocamento numa determinada rua pode depender dos fluxos das ruas transversais a ela.

Para uma formulação em função dos fluxos nos arcos as restrições do problema continuam sendo as de não negatividade e de conservação de fluxo:

$$
\begin{aligned}
& v_{a}=\sum_{r \in R} v_{a}^{r}, \quad a \in A, \\
& v_{a}^{r} \leq f_{a} w_{i}^{r}, \quad a \in A_{i}^{+}, \quad i \in I, \quad r \in R, \\
& \sum_{a \in A_{i}^{+}} v_{a}^{r}-\sum_{a \in A_{i}^{-}} v_{a}^{r}=g_{i r}, \quad i \in I, \quad r \in R, \\
& v_{a}{ }^{r} \geq 0, \quad a \in A, \quad r \in R
\end{aligned}
$$

A função objetivo não é mais a mesma da formulação com funções de custos nos arcos que dependem unicamente do fluxo no próprio arco. Para uma alocação de acordo com o ótimo do usuário, devem ser satisfeitas as condições de equilíbrio de Wardrop: as estratégias cujos custos sejam iguais ao custo ótimo (custo da melhor estratégia) terão fluxos não negativos, e aquelas cujos custos sejam maiores terão fluxos nulos, chegando à seguinte expressão:

$$
\left(X-X^{*}\right) C\left(X^{*}\right)+\left(W-W^{*}\right) \geq 0
$$

que é um problema de inequação variacional, onde $X$ representa o vetor de fluxos nos arcos, $X^{*}$ o vetor ótimo de fluxos nos arcos, $C\left(X^{*}\right)$ o vetor de custos nos arcos para os fluxos ótimos, dependente do fluxo em todos os arcos, $W$ o vetor de custos totais de espera associados ao padrão de fluxos $X$, e $W^{*}$ o vetor de custos totais de espera associados ao vetor $X^{*}$ ótimo.

Dado que o vetor de custos $C(X)$ possui um Jacobiano assimétrico, não existe uma formulação equivalente de otimização. Uma forma de resolver o problema consiste em utilizar o método da diagonalização. A cada iteração o vetor de custos é diagonalizado na solução atual, conseguindo-se desta maneira, um problema de alocação simétrica.

O método da diagonalização faz parte dos métodos de aproximação linear da função de custos $C(X)$. Pang \& Chan (1982) apresentam o seguinte algoritmo para definir a aproximação linear: 
Dado $X^{k}$ um vetor de fluxo viável, seja $X^{k+1}$ a solução do subproblema de inequações variacionais onde o vetor de custos $C\left(X^{k}\right)$ é uma aproximação de $C(X)$ no ponto $X^{k}$. Cada $C\left(X^{k}\right)$ deveria ser tal que o subproblema com o vetor de custos aproximados seja mais fácil de ser resolvido que o problema original.

Uma aproximação linear de $C(X)$ tem a forma seguinte:

$$
C^{k}(X)=C\left(X^{k}\right)+A\left(X^{k}\right)\left(X-X^{k}\right)
$$

onde $A\left(X^{k}\right)$ é uma matriz quadrada.

Se $C\left(X^{k}\right)$ for não linear, $C^{k}(X)$ será uma aproximação não linear.

Quando a matriz $A\left(X^{k}\right)$ é igual à diagonal do Jacobiano $D\left(X^{k}\right)$, o método é conhecido como Jacobiano linearizado, ou diagonalização.

Então voltando ao problema de equilíbrio, a solução do problema (43) será achada resolvendo em forma iterativa o seguinte problema:

$$
\left(X-X^{*}\right) C^{\prime}\left(X^{*}\right)+\left(W-W^{*}\right) \geq 0
$$

onde $C^{\prime}\left(X^{*}\right)$ (a aproximação linear) tem um Jacobiano diagonal.

Em Dafermos (1982) pode se apreciar que uma condição necessária e suficiente para que as funções de custos sejam fortemente monótonas, é que o Jacobiano (não necessariamente simétrico), seja definido positivo para qualquer valor de fluxo da região viável. Espera-se que esta condição seja observada na maioria das situações onde existe interação entre os fluxos dos diferentes arcos, pois geralmente o custo $c_{a}$ num arco $a$ pode depender dos fluxos em todos os arcos, mas é razoável esperar que a maior dependência seja com respeito ao fluxo do próprio arco $v_{a}$, de tal forma que $\partial c_{a} / \partial v_{a}$ seja muito maior que $\partial c_{a} / \partial v_{b}$, permitindo desprezar esta última influência.

Com tudo isso, pode-se reescrever o problema (45) como:

$$
\min \sum_{a \in A} \int_{0}^{v a} c_{a}^{\prime}(x) d x+\sum_{i \in I} \sum_{r \in R} w_{i}^{r}
$$

onde $c_{a}{ }^{\prime}(x)$ é uma componente do vetor de custos $C^{k}(X)$ que é aproximado como:

$$
C^{k}(X)=C\left(X^{k}\right)+D\left(X^{k}\right)\left(X-X^{k}\right)
$$

onde $D\left(X^{k}\right)$ é a diagonal do Jacobiano.

O problema pode ser resolvido em forma iterativa com o algoritmo apresentado na seção 4.2.

\subsection{Alocação logit nos hipercaminhos mais curtos}

No modelo de Spiess (1983), o algoritmo faz a alocação de fluxos repartindo-os nos arcos em forma proporcional às freqüências, considerando somente o conjunto de linhas atrativas. Os arcos de espera de cada linha têm uma frequiência igual à freqüência da linha associada. Os outros tipos de arcos têm frequiência infinita.

Nguyen et al. (1998) propõem a aplicação de uma forma seqüencial do modelo logit para fazer a alocação em uma rede de transporte coletivo, como uma forma de evitar a enumeração explícita de todos os hipercaminhos, mantendo as vantagens do modelo logit utilizado em redes de veículos privados. 
Neste trabalho foi implementada a alocação logit seqüencial baseada nos custos e comparada com a alocação baseada nas freqüências relativas. A primeira reparte os passageiros em uma parada entre as diferentes linhas atrativas em função dos custos de viagem desde esse ponto até o destino. A segunda reparte em função das frequiências das linhas atrativas.

O modelo logit calcula a proporção da escolha de uma determinada alternativa através da probabilidade de escolha da mesma. Pode ser aplicado a todas as etapas dos modelos de viagem, e a outros aspectos da análise de transporte e problemas urbanos. O modelo usa a seguinte fórmula:

$$
\operatorname{Prob}\left(i: A_{t}\right)=\exp \left(U_{i t}\right) / \sum_{j \in A t} \exp \left(U_{j t}\right),
$$

onde $i$ é uma alternativa que pertence ao conjunto de alternativas relevantes $A_{t}$ para um indivíduo $t$. $U_{i t}$, a utilidade da alternativa $i$, é uma função de certas características sócio-econômicas do indivíduo $t$, e das características da alternativa $i$, como tempo de viagem, custo, medida de atratividade, idade, renda, etc.

Uma forma de apresentar o modelo logit para o problema de alocação de fluxos é a seguinte:

$$
\operatorname{Prob}(p)=\exp \left(\theta V_{P}\right) / \sum_{l \in P(o . d)} \exp \left(\theta V_{l}\right), \theta<0
$$

onde $V_{P}$ é o custo associado com o caminho $p$, e $\theta$ é o coeficiente da função de utilidade, denominado aqui de parâmetro de dispersão, e $U_{i t}=\theta V_{P}$. O modelo padrão de alocação logit foi apresentado por Dial (1971). Este modelo representa uma forma viável de fazer uma alocação de equilíbrio devido a requerimentos de memória moderados para armazenar os dados da rede.

O comportamento dos motoristas de veículos é incerto, devido a que o congestionamento, a geometria das vias, as decisões dos outros motoristas, entre outros fatores, podem provocar uma dispersão na avaliação que cada viajante faz dos tempos de cada rota alternativa a seguir num determinado trajeto entre um par O-D. Os modelos probabilísticos tornam-se, então uma alternativa para tentar representar esse fenômeno.

Em vários modelos de alocação, todas as viagens entre um par origem-destino são alocadas aos arcos que compõem o caminho mais curto mediante a técnica de alocação "tudo ou nada". Uma alocação "tudo ou nada" a um único caminho contradiz o comportamento do conjunto total de viajantes, pois, muitas vezes, eles não utilizam o caminho que percebem como o mais curto $\mathrm{e}$ as alocações nestes modelos são, freqüentemente, inexatas, comprometendo as decisões dos planejadores de transportes quanto ao desenho da rede. Muitos planejadores consideram mais útil que o modelo de alocação reflita em algum grau o comportamento aparentemente não ótimo dos usuários. A qualidade das decisões dos planejadores poderia melhorar e os custos dessas diminuiriam.

Uma solução para o problema de alocação é o modelo probabilístico de Dial (1971) que evita a enumeração de caminhos. Ele aloca viagens a todos os caminhos "razoáveis" em forma simultânea, de modo tal que o efeito é idêntico ao que aconteceria se houvessem sido alocadas viagens separadamente sob certas premissas de probabilidade de escolha de caminhos.

Um caminho será razoável se ele é "eficiente". Um caminho eficiente é aquele em que o usuário não "avança para atrás". Enquanto o usuário se desloca num caminho progredindo de nó a nó, ele sempre se afasta do nó de origem e se aproxima ao nó de destino. Um caminho é 
eficiente, se cada arco contido nele tem seu nó de início mais perto do nó de origem do que seu nó final, e tem seu nó final mais perto do nó de destino do que seu nó inicial.

No que se refere à alocação logit, esta tem algumas especificações funcionais identificadas por Dial (1971) que convém ressaltar:

i) o modelo dá, a cada caminho eficiente associado a cada par O-D, uma probabilidade não nula de ser utilizado, enquanto os caminhos ineficientes têm probabilidade zero;

ii) todos os caminhos de igual custo têm igual probabilidade de uso;

iii) quando existem dois ou mais caminhos eficientes, o de menor comprimento tem maior probabilidade de uso;

iv) o modelo não enumera explicitamente os caminhos em que faz alocação; todos os caminhos eficientes para um par O-D são carregados simultaneamente;

v) o usuário do modelo pode controlar as probabilidades dos caminhos, atribuindo diferentes valores a um parâmetro de dispersão.

Uma forma seqüencial de escrever o modelo logit (49) para a escolha dos hipercaminhos baseada na probabilidade condicional proposta por Nguyen et al. (1998) é a seguinte:

$$
\operatorname{Pr}[(i, j) / i]=\exp \left(\theta\left(c_{i j}+V_{P(j)}^{\prime}\right)\right) / \sum_{\left(i, j^{\prime}\right) \in i^{+}} \exp \left(\theta\left(c_{i j^{\prime}}+V_{P\left(j^{\prime}\right)}^{\prime}\right)\right), \forall(i, j) \in i^{+}
$$

$\operatorname{Pr}[(\mathrm{i}, \mathrm{j}) / \mathrm{i}]$ é a probabilidade condicional de selecionar o $\operatorname{arco}(\mathrm{i}, \mathrm{j})$ em um hipercaminho entre o nó i e o destino.

$\boldsymbol{P}(\boldsymbol{j})$ é o conjunto de hipercaminhos que conectam o nó $j$ com o destino $d$.

$c_{i j}$ é o tempo ou custo no $\operatorname{arco}(i, j)$

$i^{+}$é o conjunto de arcos cuja cauda é o nó $i$

$V_{P(j)}^{\prime}$ é definido assim:

$$
V_{p(j)}^{\prime}=\frac{1}{\theta} \ln W_{j}
$$

onde $W_{j}$ (peso do nó $i$ ) é calculado recursivamente:

$$
W_{j}=\left\{\begin{array}{cc}
1 & \text { se } j=d \text { (nó de destino) } \\
\sum_{(j, k) \in j^{+}} \exp \left(\theta c_{j k}\right) W_{k} & \text { caso contrário }
\end{array}\right.
$$

onde $j^{+}$é o conjunto de arcos saindo do nó da rede $j$.

Para uma rede de veículos privados, onde a estrutura de custo é aditiva, ambos modelos, o global e o seqüencial geram iguais resultados. No caso de hipergrafos, onde os custos nos hipercaminhos não são funções aditivas dos custos dos arcos envolvidos, os dois modelos geram resultados diferentes, e somente o modelo seqüencial produz uma implementação eficiente que dispensa a enumeração explícita dos hipercaminhos eficientes.

Nguyen et al. (1998) consideram que devido a que o itinerário de um passageiro não depende somente da escolha dele, mas também da realização de alguns eventos externos aleatórios, como a chegada do primeiro ônibus do conjunto de linhas comuns, o mecanismo de escolha embutido no modelo seqüencial parece ser mais apropriado. Também afirmam que a 
aplicação direta do modelo logit global aos caminhos eficientes, em lugar dos hipercaminhos eficientes, destruiria a regra de repartição nos arcos de espera, embutida na abordagem dos hipercaminhos.

Em uma rede de transporte coletivo o modelo logit seqüencial levaria ao seguinte cálculo recursivo das probabilidades condicionais. A probabilidade de incluir o arco $e$ na rota dado que o nó $i$ (cauda do arco $e$ ) está incluído na rota é calculada assim:

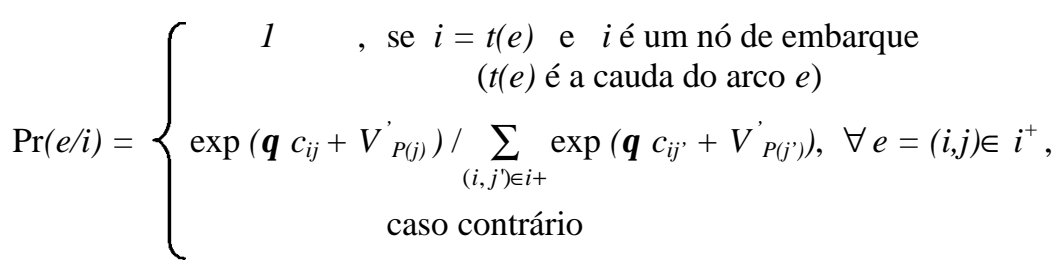

$V_{P\left(j^{\prime}\right)}^{\prime}$ é calculado como uma soma $\log$ ponderada dos pesos $W_{k}$ das cabeças dos arcos originados em j' quando j' é um nó de embarque, e como uma soma log em caso contrário.

$$
V_{P\left(j^{\prime}\right)}^{\prime}=\left\{\begin{array}{cl}
c(e)+\theta^{-1} \sum_{k \in h(e)} \alpha(e, k) \ln W_{k} & , \text { se } e \text { for arco de abordagem, } \\
\theta^{-1} \ln W_{k} & , \text { em caso contrário }
\end{array}\right.
$$

o valor do peso do nó $j, W_{j}$ é calculado recursivamente:

$$
W_{j}=\left\{\begin{array}{cl}
1 & , \text { se } j=d, \\
\exp \theta c(e) \prod_{k \in h(e)} W_{k}^{\alpha(e, k)} & , \text { se } e \text { for arco de abordagem, } \\
\sum_{(j, k) \in j^{+}} \exp \left(\theta c_{j k}\right) W_{k} & , \text { em outro caso }
\end{array}\right.
$$

onde $\alpha(e, k)$ é o coeficiente positivo da repartição de fluxo nos arcos (proporcional à frequiências relativas dos arcos incidentes).

Nguyen et al. (1998) apresentam a alocação logit seqüencial em forma de um algoritmo dividido em dois passos. No primeiro, são varridos os nós desde o destino para atrás (backward order). Aqui calcula-se o peso de cada nó $W_{j}$ e, simultaneamente, determina-se $b(e, i)$, a proporção local do nó i.

$$
b(e, i)= \begin{cases}\exp \theta c(e) \prod_{j \in h^{*}(e)} W_{j}^{\alpha(e, j)} / W_{i}, & \text { se } e \text { for arco de abordagem, } \\ \exp \theta c(e) W_{j} / W_{i} & , \text { caso contrário } e=(i, j) \in i^{+}\end{cases}
$$

No segundo passo são varridos os nós para a frente (forward order) desde a origem até o destino, alocando a demanda aos arcos eficientes. 
Estes dois passos são mostrados a continuação:

$W(d)=1$

Visite na ordem inversa topológica (backward order) e faça para cada nó $i$

Se $i$ é um nó de abordagem e $e^{\prime}=\left(i, h^{*}\left(e^{\prime}\right)\right)$

então:

$$
\begin{aligned}
& W_{i}=\exp \theta c\left(e^{\prime}\right) \prod_{j \in h^{*}\left(e^{\prime}\right)} W_{j}^{\alpha\left(e^{\prime}, j\right)} \\
& b(e, i)=1
\end{aligned}
$$

senão:

$$
\begin{aligned}
& W_{i}=\sum_{e \in i^{+}} \exp (\theta c(e)) . W_{h^{*}(e)} \\
& \text { Para cada } e \in i^{+} \text {faça } b(e, i)=\exp \theta c(e) W_{h^{*}(e)} / W_{i}
\end{aligned}
$$

O procedimento de distribuição de fluxo é o seguinte:

Inicialização

Para cada nó $i$ centróide faça $y(i)=f(i, d) \quad$ (valor do fluxo da matriz O-D)

Visite na ordem para a frente (forward order), e para cada nó $i \neq d$ faça

Para cada arco $e \in i^{+}$faça

$$
x(e)=y(i) . b(e, i)
$$

para cada $j \in h^{*}(e)$ faça

$$
y(j)=y(j)+x(e) \cdot \alpha(e, j)
$$

Na seção seguinte apresenta-se uma descrição da implementação computacional do modelo para achar a alocação de equilíbrio.

\section{Implementação computacional do problema de alocação de equilíbrio}

Nesta seção apresenta-se o algoritmo que resolve o problema da alocação de fluxo de equilíbrio para redes de transporte coletivo com congestionamento, com funções de custo assimétricas, utilizando o conceito de estratégias ou hipercaminhos. A repartição dos passageiros nos diferentes caminhos elementares de cada hipercaminho pode ser feita utilizando dois métodos que serão apresentados e brevemente comparados, na seção 6 . Tratase de um modelo logit, e outro, utilizando uma repartição dos fluxos entre os caminhos elementares de cada hipercaminho, em forma proporcional às freqüências das linhas.

Nesta implementação as funções de custos nos arcos são funções monótonas não simétricas dos fluxos nos arcos. Como parte da solução, foi implementado um método de diagonalização baseado no Jacobiano do vetor de custos, que é um procedimento iterativo em que as funções de custo são aproximadas em forma linear na solução corrente, obtendo-se um novo problema com funções de custo simétricas. Será utilizado um procedimento para resolver o problema de alocação em uma rede de transporte coletivo baseada no algoritmo de Frank-Wolfe (1956). De Cea \& Fernández (1993) indicam que as condições de monotonicidade das funções de custo são suficientes mas não necessárias em algoritmos de 
diagonalização, que na prática apresentam excelentes propriedades de convergência, mesmo até quando as condições não são satisfeitas.

Como as restrições de conservação de fluxo e de não negatividade determinam um conjunto convexo, então o problema pode ser resolvido por métodos iterativos para resolver problemas de inequação variacional (ver Pang \& Chang, 1982; Parada, 1989), em particular, pelo procedimento baseado no método de Frank-Wolfe, que não requer guardar os volumes para cada destino em forma explícita.

A continuação apresenta-se o algoritmo proposto para resolver o problema de equilíbrio, no qual é utilizada uma adaptação do método de Frank-Wolfe, que resolve o problema para o caso com congestionamento do problema linearizado, e faz uma alocação logit:

\subsection{Algoritmo de solução}

0. Inicialização

Gere a rede aumentada

Ache uma solução viável $\left(v^{0}, w^{0}\right)$

Seja $l=0$

1. Seja $l=l+1$

Ache os hipercaminhos mais curtos para todos os pares O-D

Faça uma alocação logit seqüencial e ache $\left(v^{\prime}, w^{\prime}\right)$ aplicando os algoritmos de repartição local e distribuição de fluxo com os custos nos $\operatorname{arcos} t_{i}=t_{i}\left(v^{l-1}\right)$

2. Calcule o tamanho de passo $\lambda^{l}$ que minimize a função objetivo no segmento de linha determinado por: $(1-\lambda) \cdot\left(v^{l-1}, w^{l-1}\right)+\lambda .\left(v^{\prime}, w^{\prime}\right), \quad 0 \leq \lambda \leq 1$

3. Atualize $\left(v^{l}, w^{l}\right)=\left(1-\lambda^{l}\right) \cdot\left(v^{l-1}, w^{l-1}\right)+\lambda^{l} \cdot\left(v^{\prime}, w^{\prime}\right)$

Se $\left\|\left(v^{l}, w^{l}\right)-\left(v^{l-1}, w^{l-1}\right)\right\|<\varepsilon$ então pare, senão vá ao passo 1

As principais rotinas desta implementação podem ser identificadas como:

- a geração da rede de transporte coletivo (rede aumentada),

- a determinação das rotas, representados pelos hipercaminhos,

- a alocação dos passageiros nessas rotas.

Para a geração da rede aumentada parte-se da representação da rede viária, feita através de um grafo $\mathrm{G}=(\mathrm{N}, \mathrm{A})$, onde $\mathrm{N}$ é o conjunto de nós e $\mathrm{A}$ é o conjunto de arcos. A região em estudo é dividida em zonas de tráfego. Cada uma destas tem um centróide, onde é suposto que está concentrada a demanda de passageiros. Uma matriz de viagens origem-destino representa os deslocamentos de passageiros entre cada par de centróides. Os nós da rede representam centróides, paradas e interseções das vias. Os arcos representam as vias, conectando paradas consecutivas, e também existem conectores (arcos de caminhada) que representam os acessos dos centróides à rede viária. A rede "aumentada" é obtida pela adição de arcos de caminhada, utilizados para o acesso e saída da rede e transferências entre linhas. Além disto, cada uma das linhas disponíveis entre duas paradas consecutivas, i e j, é representada por um arco individual a. Isto é, duas linhas diferentes entre um mesmo par de nós são representadas por dois arcos diferentes. Na rede aumentada, cada parada de ônibus atendida por $k$ linhas, é representada por $k+1$ nós diferentes (um da parada própria e um nó adicional para cada linha). A geração da rede aumentada explode o tamanho da rede original, 
tanto em número de arcos como em número de nós. Uma das contribuições desta pesquisa foi o desenvolvimento de um procedimento para a geração automática da rede aumentada (Castro \& Leal, 1998).

Para a determinação dos hipercaminhos foi utilizada uma sub-rotina que se encarrega de encontrar os custos totais de viagem desde todos os nós até um nó que é fixado como destino, para custos fixos nos arcos. Os valores dos custos são fixos em função dos fluxos de uma iteração anterior. Na primeira iteração parte-se de um conjunto de fluxos viáveis, podendo ser nulos.

As funções de custos nos arcos que foram utilizadas nesta implementação, dependem somente dos fluxos de dois arcos, ou seja, além do fluxo do próprio arco, depende do fluxo de um outro arco da rede. Como a atualização dos custos é feita a cada iteração, para este caso particular, em que as funções de custo nos arcos dependem somente de dois arcos, foi conveniente identificar esse outro arco, cujo fluxo influi no custo. Foi criada uma sub-rotina para identificação desse arco auxiliar no cálculo do custo, o que agiliza a atualização dos custos. Para cada arco de espera, foi considerado que o custo de espera depende do número de passageiros esperando na parada e da quantidade de passageiros que já está dentro do veículo, quando este chega na parada. Para os arcos em veículo, foi considerado que o custo depende do número de passageiros que já está dentro dele e dos que acabam de subir. Nesta implementação, foi utilizado o algoritmo de Spiess para custos fixos, detalhado na seção 4.1, que usa etiquetas nos arcos que correspondem às linhas consideradas atrativas pelos passageiros. No passo 1 deste algoritmo, para poder escolher o arco $a=(i, j)$ com o menor $\mathrm{u}_{\mathrm{j}}{ }^{+} \mathrm{ca}$, os arcos devem ser ordenados em forma crescente, segundo a etiqueta $\mathrm{u}_{\mathrm{j}}{ }^{+} \mathrm{ca}$. Para isto foi utilizada uma estrutura heap binária.

A alocação de passageiros foi realizada neste trabalho usando dois métodos: Um que reparte os fluxos nas paradas atendidas por mais de uma linha atrativa, em forma proporcional às freqüências das respectivas linhas e outro que efetua a repartição segundo uma alocação logit.

O tamanho do passo corresponde ao valor de ë que minimiza a função objetivo do problema linearizado, para dois valores de fluxos calculados em duas iterações seguidas. Uma forma de calcular este valor, é igualando a zero a derivada da função objetivo para um valor do fluxo que depende de ë. Deve se verificar que o valor achado corresponda a um mínimo e não a um máximo, já que a derivada nula não garante que seja o mínimo. Para a igualação a zero da derivada foi implementado um cálculo iterativo utilizando o método da bi-seção, com valores iniciais de ë entre 0 e 1 . Além disso, a cada iteração, tem que ser examinadas as condições nos extremos do intervalo de busca. Deve-se verificar, por exemplo, a condição de que as pendentes da função a ser minimizada nos extremos do intervalo de busca tenham sinais diferentes para garantir que pode haver um mínimo em algum ponto do intervalo de busca. No caso em que as pendentes nos extremos do intervalo de busca têm o mesmo sinal o mínimo acontece em um dos extremos do intervalo de busca. Para identificar em qual dos extremos se dá o valor mínimo, basta avaliar a função nos dois pontos, e comparar estes valores.

Estas rotinas foram implementadas em linguagem Delphi e o programa foi aplicado na rede de transporte coletivo da cidade do Rio de Janeiro, com resultados resumidos a seguir. 


\section{Resultados da aplicação da alocação de equilíbrio}

Nesta seção são apresentados os primeiros resultados da aplicação da implementação computacional. Testes foram feitos previamente com redes utilizadas nos artigos de Spiess (1983) e Wu et al. (1994) produzindo resultados corretos.

Os dados utilizados nesta aplicação correspondem à Região Metropolitana do Rio de Janeiro. Esta região era atendida, na época da construção da base de dados, por um total de 605 linhas de ônibus, das quais 328 eram linhas municipais e 277 eram linhas intermunicipais. Também são consideradas as duas linhas de metrô: linha 1: Botafogo-Saens Peña, e linha 2: EstácioEngenho da Rainha. Não foi considerado o ramal Copacabana-Botafogo da linha 1 do metrô, pois os dados utilizados são anteriores ao funcionamento daquele trecho. Além das linhas de ônibus e das de metrô, também são considerados 10 ramais de trem e 5 linhas de barcas e aerobarcas nas rotas Rio-Niterói, Rio-Ilha do Governador e Rio-Ilha de Paquetá.

A seguir descrevem-se os dados gerais das linhas dos diversos modos de transporte coletivo consideradas nesta aplicação.

ÔNIBUS: para este modo foram codificadas 605 linhas.

METRÔ: foram codificadas as duas linhas.

TREM: foram codificados 10 ramais: ramal Deodoro, Santa Cruz, Japeri, Paracambi, Belford Roxo, São Mateus, Gramacho, Vila Inhomirim, Guapimirim e o ramal de Niterói.

BARCAS / AEROBARCOS: foram codificadas 5 linhas, sendo 3 do modo barca, a saber: Rio-Niterói, Rio-Ilha do Governador e Rio-Ilha de Paquetá. Para o modo aerobarco foram codificadas as linhas Rio-Niterói e Rio- Ilha de Paquetá.

A rede viária básica contém 1098 nós e 2753 arcos. A rede generalizada contém 30641 nós e 85341 arcos.

A região em estudo está dividida em 94 zonas de tráfego e parte-se da hipótese de que a demanda de passageiros está concentrada nos centróides de cada zona. Os valores das quantidades de viagens para cada par de zonas origem-destino estão contidos em matrizes de origem destino (matrizes O-D). São duas matrizes O-D, uma para o período de pico da manhã, e a outra para a tarde.

Foi aplicado o algoritmo, descrito na seção 5.1, aos dados das matrizes origem-destino das horas pico da manhã e da tarde.

As funções de custos nos arcos que foram utilizadas neste estudo são teóricas uma vez que a criação destas funções deveria surgir a partir de um estudo prévio que escapa ao escopo desta pesquisa. Estas funções de custos nos arcos deveriam ser criadas de forma tal que refletissem o padrão de fluxos observados, além de observar as características de monotonicidade e convexidade para garantir a convergência e existência da solução do problema.

Consideraram-se 4 tipos de arcos sendo aqui especificada uma função de custo para cada tipo de arco:

$c_{a}(v)=\alpha_{1} t_{a}$ para arcos de caminhada

$c_{a}(v)=\alpha_{2}\left(\left(v_{b}+\beta_{2} v_{d}\right) / K\right)^{\rho}$ para arcos de espera

$c_{a}(v)=\alpha_{3} t_{a}+\beta_{3}\left(\left(v_{d}+\gamma_{3} v_{b}\right) / K\right)^{\rho}$ para arcos em veículo 
$c_{a}(v)=\alpha_{4} t_{a}$ para arcos de desembarque

onde $t_{a}$ é o tempo de deslocamento no $\operatorname{arco}, v$ é o fluxo no arco $a, v_{b}$ e $v_{d}$ são os fluxos nos arcos em veículo e arco de espera prévios ao arco corrente $a$, respectivamente. As variáveis $\alpha_{1}, \alpha_{2}, \alpha_{3}, \alpha_{4}, \beta_{1}, \beta_{2}, \gamma_{3}$ e $\rho$ são parâmetros das funções de custos.

Foram considerados dois critérios de parada para a execução do programa. Um deles é baseado na função GAP definida como: $G a p(h)=\min S(h)^{T}(h-x)$ onde $x$ é um fluxo qualquer na região viável, e $\mathrm{h}$ é o valor corrente do fluxo. O segundo critério é fixar o número de iterações. Os valores default sugeridos pelo manual do pacote de EMME/2 são de 15 iterações e um GAP relativo de $0.5 \%$ ou 0.005 . O GAP relativo é obtido dividindo o valor do GAP absoluto pelo valor do custo ou tempo total na rede.

O critério de parada atingido foi geralmente o número de iterações previamente estabelecido no programa, o qual foi fixado em 10 ou 20 iterações. Em termos de tempo de CPU, utilizando uma máquina Pentium 100 a média foi de 37 minutos para 20 iterações, o que dava um valor médio de um minuto e 50 segundos por iteração. Isto depende muito da estrutura particular da rede, dos algoritmos utilizados e das estruturas de dados utilizadas. No caso, a rede da Região Metropolitana do Rio de Janeiro apresenta alta concentração de linhas em determinadas zonas de tráfego da cidade, como por exemplo no centro da cidade na avenida Rio Branco, na avenida Brasil, na avenida Nossa Senhora de Copacabana. Em média cada linha passava por 10 pontos de parada, e cada ponto de parada era atendido por 20 linhas de transporte coletivo. Aqui deve se levar em conta que as linhas com percurso de ida e volta foram consideradas como duas linhas independentes e que alguns nós destas linhas faziam parte tanto do percurso de ida quanto da volta.

O uso do algoritmo de Frank-Wolfe produz bons resultados mas sem a precisão que poderiam atingir outros métodos. Pompermayer (1997) indica que o método de Frank-Wolfe mesmo considerando estas restrições, ainda é um dos mais utilizados em aplicações de transportes, devido às incertezas envolvidas no levantamento dos dados, o que torna inadequado preocupar-se com a exatidão na busca do ótimo.

A continuação, apresenta-se uma tabela com os resultados das alocações efetuadas para distribuição dos fluxos proporcionais às freqüências e segundo o modelo logit. Mostram-se os valores da função objetivo e os gaps relativos para 20 iterações.

Tabela 6.1 - Valores obtidos para a Função Objetivo e o GAP em 20 iterações

\begin{tabular}{|l|r|r|r|r|}
\hline ITERAÇÃO & \multicolumn{1}{|c|}{ LOGIT } & GAP RELATIVO & PROP. A FREQÜÊNCIA & GAP RELATIVO \\
\hline 1 & $17.899 .024 .598,88$ & & $17.842 .842 .728,40$ & \\
\hline 2 & $24.550 .627 .095,93$ & 0,27093412 & $25.907 .422 .572,91$ & 0,31128453 \\
\hline 3 & $26.687 .245 .954,47$ & 0,08006142 & $21.961 .264 .503,82$ & $-0,17968720$ \\
\hline 4 & $19.625 .430 .975,53$ & $-0,35982980$ & $19.588 .143 .131,77$ & $-0,12115091$ \\
\hline 5 & $20.089 .696 .986,07$ & 0,02310966 & $19.038 .585 .555,35$ & $-0,02886546$ \\
\hline 6 & $18.029 .863 .897,53$ & $-0,11424563$ & $19.261 .889 .536,68$ & 0,01159305 \\
\hline 7 & $17.993 .223 .210,94$ & $-0,00203636$ & $18.705 .150 .163,70$ & $-0,02976396$ \\
\hline 8 & $17.046 .234 .637,82$ & $-0,05555412$ & $19.025 .129 .991,95$ & 0,01681880 \\
\hline 9 & $16.842 .456 .179,44$ & $-0,01209909$ & $17.854 .737 .268,10$ & $-0,06555082$ \\
\hline 10 & $17.225 .718 .016,10$ & 0,02224940 & $19.221 .815 .558,28$ & 0,07112118 \\
\hline 11 & $17.153 .654 .808,02$ & $-0,00420104$ & $17.856 .844 .543,78$ & $-0,07643965$ \\
\hline 12 & $16.488 .412 .103,52$ & $-0,04034607$ & $19.081 .601 .380,44$ & 0,06418522 \\
\hline 13 & $17.765 .321 .903,90$ & 0,07187654 & $17.849 .812 .947,70$ & $-0,06900848$ \\
\hline
\end{tabular}




\begin{tabular}{|r|r|r|r|r|}
\hline 14 & $16.648 .129 .487,66$ & $-0,06710618$ & $18.805 .585 .815,17$ & 0,05082388 \\
\hline 15 & $16.844 .991 .746,64$ & 0,01168669 & $18.097 .120 .000,90$ & $-0,03914799$ \\
\hline 16 & $16.630 .216 .234,25$ & $-0,01291478$ & $17.811 .652 .761,26$ & $-0,01602699$ \\
\hline 17 & $16.658 .930 .101,34$ & 0,00172363 & $17.935 .295 .432,08$ & 0,00689382 \\
\hline 18 & $16.642 .968 .825,54$ & $-0,00095904$ & $18.823 .955 .041,80$ & 0,04720897 \\
\hline 19 & $16.896 .827 .762,81$ & 0,01502406 & $18.929 .347 .512,97$ & 0,00556768 \\
\hline 20 & $16.485 .869 .390,58$ & $-0,02492792$ & $18.849 .089 .046,97$ & $-0,00425795$ \\
\hline
\end{tabular}

$\mathrm{Na}$ tabela anterior pode-se observar como variam os valores da função objetivo para as duas formas de alocar os passageiros nos arcos da estratégia ótima. A primeira alocação é feita em função de um modelo logit que reparte os fluxos nos arcos em função de parâmetros que dependem da distância do ponto analisado até o nó de destino. A segunda alocação é em função das freqüências dos arcos que representam as linhas de ônibus.

Pode-se notar que os valores observados da função objetivo oscilam em torno a um mínimo local e em algumas iterações, ficam até pior que na solução da iteração prévia o que pode ser considerado uma característica do Frank e Wolfe. O procedimento usando a alocação Logit teve, neste experimento, um desempenho um pouco melhor, com o valor mínimo alcançado na 20. iteração. Seu valor mínimo ficou $8 \%$ abaixo do valor mínimo obtido na alocação proporcional à freqüência, alcançado na 16. iteração.

\section{Conclusões}

Nesta pesquisa foi desenvolvido e implementado um modelo para alocação de equilíbrio em uma rede de grande porte. Foram apresentados os fundamentos teóricos do modelo e aspectos da implementação computacional.

Para aplicações práticas é necessário um estudo de fluxo que determine as funções de custos nos arcos adequadas à região em estudo, para se obter valores de fluxos que possam ajudar a simular uma situação real e auxiliar na tomada de decisões ao planejador do sistema de transporte coletivo. A modelagem da função de custo merece ser objeto de estudos adicionais. Acredita-se que, dentro de um escopo de um estudo completo de tráfego na região em estudo, podem ser calibradas funções de custo próprias da cidade estudada, em função da geometria particular da rede, do número de linhas, etc., que expliquem, em melhor forma, os padrões de fluxo observados em uma situação real.

Considerando o tamanho da rede analisada e os tempos de CPU, pode-se concluir que os tempos de processamento obtidos são aceitáveis. De fato, redes mais detalhadas consomem maior tempo nos cálculos computacionais, e deve-se chegar em uma solução de compromisso, onde os critérios em conflito seriam a memória necessária para redes mais detalhadas e o tempo de computação. O nível de detalhe da representação da rede de transporte coletivo deverá ser escolhido pelos planejadores de acordo com os objetivos do estudo.

Uma alocação logit leva em consideração uma forma de distribuição dos fluxos mais realista, em relação ao comportamento dos usuários do sistema de transporte público, do que uma distribuição proporcional às frequiências das linhas. Mas, nos casos em que não existe congestionamento, é possível supor que os passageiros serão distribuídos entre as linhas consideradas comuns, ou atrativas, em forma proporcional às freqüências. 
Em uma rede em que os transbordos são permitidos podem aparecer rotas que contenham um número absurdo de transbordos. Isto não refletiria a realidade da maioria das cidades de países em desenvolvimento, em que a maioria dos usuários do sistema de transporte coletivo é considerada como usuários cativos, os quais procuram minimizar o custo monetário da viagem independentemente do tempo de viagem. Pode ser sugerido algum tipo de penalidade que limite o número de transbordos permitidos, o qual refletiria melhor uma situação real e diminuiria o tempo de cálculo dos algoritmos.

$\mathrm{Na}$ aplicação relacionada a este estudo apresentam-se apenas valores dos custos totais de uma alocação de equilíbrio na rede do Rio de Janeiro. Algumas sugestões para os planejadores do sistema de transporte podem surgir a partir dos resultados obtidos, ao se analisar os dados de uma forma desagregada. Para tal é necessário dispor de um método de visualização associado a um sistema de informações geográficas para poder interpretar a massa de dados relacionados aos arcos e trechos de linhas. O modelo está sendo implementado nesta direção, através de interfaces com o Transcad. A partir daí, podem ser analisados fluxos e, uma vez verificados trechos com engarrafamentos, realizar alguns experimentos. Por exemplo, pode-se mudar o tamanho dos veículos de algumas linhas segundo os fluxos observados, mudar as freqüências, mudar os itinerários de algumas linhas, tirar linhas, etc. Os primeiros resultados da pesquisa são encorajadores no sentido de se dispor de um procedimento avançado de análise de redes de transporte público em cidades de grande porte.

\section{Agradecimentos}

Os autores agradecem ao $\mathrm{CNPq}$ o apoio dado à pesquisa. $\mathrm{O}$ primeiro autor foi bolsista de doutorado e o segundo recebeu bolsa de produtividade de pesquisa.

\section{Referências Bibliográficas}

(1) Ahuja, R.K.; Magnanti, T.L. \& Orlin, J.B. (1989). Network Flows. In: Handbooks in Operations Research and Management Science, vol. 1 - Optimization [edited by G.L. Nemhauser, A.H.G. Rinnooy Kan and M.J. Todd], 211-369.

(2) Andréasson, I. (1976). A Method for the Analysis of Transit Networks. Second European Congress on Operations Research, Amsterdam, North-Holland.

(3) Bazaraa, M.S. (1990). Nonlinear Programming: Theory and Algorithms. John Wiley \& Sons, New York.

(4) Bouzaïene-Ayari, B.; Gendrau, M. \& Nguyen, S. (1995). An Equilibrium-Fixed Point Model for Passenger Assignment in Congested Transit Networks. Publication 1001, Centre de Recherche sur les Transports, Université de Montréal.

(5) Bunster, J.P. (1986). Tratamiento de líneas comunes en modelos de asignación de viajes a redes de transporte público. Dissertação de Mestrado em Ciências da Engenharia, Escuela de Ingeniería, Pontificia Universidad Católica de Chile.

(6) Castro A., F.R. \& Leal, J.E. (1997). Comparação de duas abordagens para o problema de alocação de equilíbrio para redes de transporte público. Anais do XI ANPET, 1, 314-326. 
(7) Castro A., F.R. \& Leal, J.E. (1998). Redefinição automática da rede de transporte coletivo para alocação de fluxo de equilíbrio. Transportes, 6(2), 28-45.

(8) Chriqui, C. (1974). Rèseau de Transport en Commun: Les Problémes de Cheminement et d'Accés. Ph. D. thesis, Dép. I.R.O. Université de Montréal, Canadá.

(9) Chriqui, C. (1975). Public transit network assignment method. Publication 21, Centre de recherche sur les transports, Université de Montréal, Canadá.

(10) Chriqui, C. \& Robillard, P. (1975). Common bus lines. Transportation Science, 9, 115121.

(11) Dafermos, S. (1980). Traffic equilibrium and variational inequalities. Transportation Science, 14, 42-54.

(12) De Cea, J.; Fernández, J.E. \& Crovetto, G. (1990). Análisis comparativo de tres modelos de asignación a redes de transporte público basados en el concepto de ruta. Apuntes de Ingeniería, 39, 5-22.

(13) De Cea, J. \& Fernández, E. (1993-I). Comportamiento de los viajeros y modelos de asignación de equilibrio en redes de transporte público. Apuntes de Ingeniería, 50, $5-20$.

(14) De Cea, J. \& Fernández, E. (1993-II). Transit Assignment for Congested Public Transport Systems: An Equilibrium Model. Transportation Science, 27(2), 133-147.

(15) Dial, R.B. (1967). Transit Pathfinder Algorithm. Highway Research Record, 205, 67-85.

(16) Dial, R.B. (1971). A Probabilistic Multipath Traffic Assignment Model which obviates Path Enumeration. Transportation Research, 5, 83-111.

(17) Dijkstra, E.W. (1959). A note on two problems in connection with graphs. Numeriche Matematik, 1, 269-271.

(18) Frank, M. \& Wolfe, P. (1956). An Algorithm for Quadratic Programming. Naval Research Logistics Quarterly, 3, 95-110.

(19) Gendrau, M. (1984). Étude approfondie d'un modèle d'équilibre pour l'affectation des passagers dans les réseaux de transport en commun. Publication 384, Centre de recherche sur les transports, Université de Montréal, Canadá.

(20) Hasselströem, D. (1981). Public Transportation Planning - A Mathematical Programming Approach. Ph. D Thesis, Department of Business Administration, University of Gothenburg, Sweden.

(21) Knoppers, P. \& Muller, T. (1995). Optimized Transfer Opportunities in Public Transport. Transportation Science, 29(1), 101-105.

(22) Kirchhoff, P. (1995). Public transit research and developing in Germany. Transportation Research A, 29(1), 1-7.

(23) Lampkin, W. \& Saalmans, P.D. (1967). The Design of Routes, Service Frequencies and Schedules for a Municipal Bus Undertaking: A case study. Operation Research Quarterly, 18, 375-397. 
(24) Leal, J.E. \& Pompermayer, F.M. (1997). Experiências com o tamanho de passo no algoritmo de Frank-Wolfe para redes de transportes. Anais do XI ANPET, 1, 327-336.

(25) Mackett, R.L. \& Edwards, M. (1998). The impact of new urban public transport systems: will the expectations be met? Transportation Research A, 32(4), 231-245.

(26) Mandl, C.E. (1978). Algorithms and computer programs in deterministic network optimization applied to public systems. Research Memorandum n. 126, Institute for Advanced Studies, Viena.

(27) Marguier, P.H.J. \& Ceder, A. (1984). Passenger Waiting Strategies for Overlapping Bus Routes. Transportation Science, 18(3), 207-230.

(28) Moore, E.F. (1957). The Shortest Path through a maze. International Symposium on the Theory of Switching Proceedings, Harvard University.

(29) Nguyen, S. (1984). An Algorithm for the Traffic Assignment Problem. Transportation Science, 18(2), 203-216.

(30) Nguyen, S. \& Dupuis, C. (1984). An Efficient Method for Computing Traffic Equilibria in Networks with Asymmetric Transportation Costs. Transportation Science, 18(2), 185-202.

(31) Nguyen, S. \& Pallottino, S. (1986). Hyperpaths and Shortest Hyperpaths. Combinatorial Optimization, Lectures Notes in Mathematics, 1403, 258-271, Springer-Verlag.

(32) Nguyen, S. \& Pallottino, S. (1988). Equilibrium traffic assignment for large scale transit networks. Publication 494, Centre de Recherche sur les Transports, Université de Montréal.

(33) Nguyen, S.; Pallottino, S. \& Malucelli, F. (1994). A Modeling Framework for the Passenger Assignment on a Transport Network with Time-Tables. Publication 94-47, Centre de Recherche sur les Transports, Université de Montréal.

(34) Nguyen, S.; Pallottino, S. \& Gendrau, M. (1998). Implicit Enumeration of Hyperpaths in a Logit Model for Transit Networks. Transportation Science, 32(1), 54-64.

(35) Pallottino, S.; Nguyen, S. \& Marcotte, P. (1995). On the Existence of Equilibrium Flows for Transit Networks. Institute for Operations Research and the Management Sciences.

(36) Pang, J.S. \& Chan, D. (1982). Iterative Methods for Variational and Complementarity Problems. Mathematical Programming, 24, 284-313.

(37) Parada D., V.M. (1989). Algoritmos para problemas de equilíbrio em redes. Tese de Doutorado em Engenharia de Sistemas de Computação, COPPE, UFRJ.

(38) Pietrantonio, H. (1997). Modelos Normativos em Transportes Urbanos: Aplicação ao Projeto de Redes de Transporte Público Coletivo Regular Urbano de Passageiros TPCR/UP. Tese de Doutorado, Escola Politécnica da USP.

(39) Pompermayer, F.M. (1997). Sistema para alocação de fluxos multimodal multiproduto para transporte de carga. Dissertação de Mestrado, Departamento de Engenharia Industrial, Pontifícia Universidade Católica do Rio de Janeiro. 
(40) Ramanathan, B.; Jayakrishnan, R. \& Mcnally, M.G. (1995). Assignment of Transit Ridership Under Information Supply. Institute for Operations Research and the Management Science.

(41) Robillard, P. (1971). 0-1 Hyperbolic Programming Problems. Naval Research Logistic Quarterly, 18, 47-57.

(42) Rossetti, M.D. \& Turitto, T. (1998). Comparing static and dynamic threshold based control strategies. Transportation Research A, 32(8), 607-620.

(43) Spiess, H. (1983). On optimal route choice strategies in transit networks. Publication 286, Centre de Recherch sur les Transports, Université de Montréal.

(44) Spiess, H. (1984). Contributions à la Théorie et aux Outils de Planification des Réseaux de Transports en Commun. Université de Montréal.

(45) Spiess, H. \& Florian, M. (1989). Optimal strategies: A new assignment model for transit networks. Transportation Research B, 23(2), 83-102.

(46) Suwansirikul, C.; Friesz, T.L. \& Tobin, R.L. (1987). Equilibrium network design problem. Transportation Science, 21(4).

(47) Tertoolen, G.; Van Kreveld, D. \& Verstraten, B. (1998). Psychological resistance against attempts to reduce private car use. Transportation Research A, 32(3), 171-181.

(48) Tisato, P. (1998). Service unreliability and bus subsidy. Transportation Research A, 32(6), 423-436.

(49) Torres G., L.E. (1987). Contribuição ao problema de equilíbrio em redes. Tese de Doutorado em Engenharia de Sistemas de Computação, COPPE, UFRJ.

(50) Voss, S. \& Daduna, J.R. (1995). Customer Orientation in Public Mass Transit through Passenger Information Systems. Institute for Operations Research and the Management Science.

(51) Wu, J.H.; Florian, M. \& Marcotte, P. (1994). Transit equilibrium assignment: a model and solution algorithms. Transportation Science, 28(3).

(52) Zhan, F.B. \& Noon, C.E. (1998). Shortest Paths Algorithms: An Evaluation using Real Road Networks. Transportation Science, 32(1), 65-73. 